\title{
Update on Pseudotumor Cerebri (Idiopathic Intracranial Hypertension)
}

\section{(ㄷ) (i) (우)}

Authors

Jan Hoffmann, Arne May

\section{Affiliation \\ Department of Systems Neuroscience, University Medical Center Hamburg-Eppendorf, Hamburg}

Key words

pseudotumor cerebri, idiopathic intracranial hypertension, headache

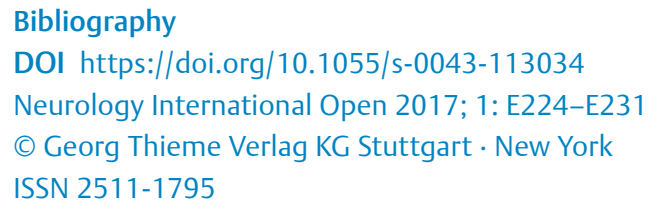

Correspondence

PD Dr. med. Jan Hoffmann

Department of Systems Neuroscience

University Medical Center Hamburg-Eppendorf

Martinistraße 52

20246 Hamburg

j-r.hoffmann@uke.de

\section{ABSTRACT}

Idiopathic intracranial hypertension is characterized by an increase of intracranial pressure of unknown etiology. The clinical presentation is dominated by progressive visual disturbances, which are commonly the result of a papilledema, headache and cranial nerve palsies. Clinical studies have revealed over the past years that the syndrome may also be associated with olfactory disturbances, cognitive deficits and a pulsatile tinnitus. The underlying pathomechanism is probably based on a disturbance of venous outflow, which causes a reduced absorption of cerebrospinal fluid that results in an increase of cerebrospinal fluid pressure. It remains unclear if the venous outflow disturbance results from the overweight-induced increase of intrathoracic pressure, the presence of sinus vein stenoses or both conditions. Recently hormonal factors have also been discussed, but the mechanisms behind a potential influence on intracranial pressure remain unclear.

Treatment of idiopathic intracranial hypertension relies mainly on an effective body weight reduction and medication with carboanhydrase inhibitors, which can reduce the production of cerebrospinal fluid and body weight. Results of the first randomized, placebo-controlled trial as well as a 12-month follow-up show that acetazolamide effectively reduces papilledema, visual disturbances and headache in idiopathic intracranial hypertension. In contrast, the evidence for the carboanhydrase inhibitors topiramate and furosemide still relies on open-label trials, because double-blind, placebo-controlled trials for their efficacy in idiopathic intracranial hypertension do not exist. In addition to the treatment with carboanhydrase inhibitors, increasing evidence suggests that the somatostatin analog octreotide may be effective in the treatment of idiopathic intracranial hypertension, but to date no randomized, double-blind, placebo-controlled trials exist to confirm this observation.

\section{Introduction}

In 1904 the German neurologist Max Nonne introduced the term pseudotumor cerebri (PTC) to describe the clinical syndrome of chronically elevated intracranial pressure of unknown etiology. As diagnostic options grew broader and more effective over time, however, numerous pathological conditions that can cause chronically high intracranial pressure were identified, which occasioned its classification into a primary and secondary form.

For years the term pseudotumor cerebri has been used as an umbrella term for chronic intracranial hypertension without further specifying its underlying cause. Pseudotumor cerebri has a primary form, idiopathic intracranial hypertension (IIH), and various secondary forms that can co-occur with other diseases, such as cerebral venous sinus thrombosis or hormonal changes[1, 2]. The following review is limited to the primary form of PTC.

The prevalence of IIH is 8.6 cases per 100000 in the general population $[3,4]$, although the number is probably much higher considering that existing epidemiological studies are several decades old and apply different diagnostic criteria. Moreover, because the clinical presentation of IIH can be so similar to other primary headache disorders such as migraine or tension-type headache, especially their chronic forms, the disease may be underdiagnosed $[5,6]$. Furthermore, most persons suffering from IIH are overweight $[7,8]$; against the backdrop of increasing worldwide obesity, the 
prevalence of IIH is also assumed to be growing [9]. In approximately $90 \%$ of adult cases, IIH affects women of reproductive age [10], although the relationship to gender is largely unclear. No such gender-specific differences have been observed in children suffering from IIH, especially young, prepubertal children [11-13].

\section{Pathophysiology}

The production and absorption of cerebrospinal fluid (CSF) is finely balanced. IIH was initially thought to be caused by an overproduction of CSF, a belief now largely refuted, which is why current research is focused on the absorption of CSF and venous drainage.

In IIH, patients are usually very obese, intracranial pressure is clearly correlated with BMI, and weight loss is the most effective treatment option. These facts suggest that obesity-related high intra-abdominal and intrathoracic pressure increases cerebral venous pressure and reduces the absorption of CSF, ultimately causing intracranial pressure to rise. Even as plausible as this hypothesis may seem, several questions remain to be answered. It does not explain female preponderance, especially because women, unlike men, tend to accumulate fat around the hips rather than the abdomen. Moreover, the majority of obese women do not suffer from IIH, and unlike adults, children with IIH are usually not at all overweight. Obesity alone can therefore not sufficiently explain the underlying pathomechanism of IIH [13].

This has led to speculation over the last several years that various hormonal factors may be the link between obesity and IIH. Therefore, the role of hormonal processes that occur in adipose tissue receive increasing attention $[14,15]$. In IIH patients, for example, the serum leptin concentration is significantly higher than in obese control subjects, pointing the discussion to central leptin resistance $[16,17]$. Interestingly, leptin concentration in the affected patients is higher in women than in men, and hence this mechanism might explain why women are particularly affected by IIH. It is also known that sex hormones, especially androgen, are markedly altered in IIH patients. Because its availability is regulated by 11 ß-hydroxysteroid dehydrogenase type 1 (11ß-HSD1), the potential role of glucocorticoids, especially cortisol, is also discussed. 11 ß-HSD is especially active in adipose tissue and increased activity boosts cortisol production. Cortisol's effect on glucocorticoid and mineralocorticoid receptors could increase the production of CSF [15]. This mechanism could not only explain the development of intracranial hypertension but also the female preponderance. However, current data does not suffice to draw specific pathophysiological conclusions.

Another hypothesis posits that one or more transverse sinus stenoses (TSS) impede venous drainage, and like the previously described mechanism, cause intracranial pressure to rise. In support of this hypothesis, TSS was identified in approximately $90 \%$ of adult IIH patients, and in cases of bilateral TSS, endovascular treatment of the stenoses may alleviate or eliminate IIH [18-20]. In contrast, hemodynamic models show that only bilateral TSS are able to block drainage enough to significantly impair CSF absorption [21]. Neither can this mechanism explain why predominantly women are affected by $\mathrm{IIH}$. It is also far from clear whether TSS are primary or secondary, i. e., from external compression due to elevated intracranial pressure, because TSS in IIH are usually very prolonged and in some cases remit after pressure is relieved. If the genesis is secondary, TSS may still induce a vicious circle: high intracranial pressure could cause TSS, which could trigger or worsen a drainage block, thus impairing CSF absorption even further and thereby increasing intracranial pressure. Ultimately this would lead to a further secondary increase in TSS and could further aggravate the clinical picture. Because endovascular treatment could break this vicious circle, its efficacy cannot help to differentiate between primary and secondary TSS.

\section{Clinical Presentation and Diagnostics}

Due to increasing diagnostic options, especially imaging procedures, diagnostic criteria for IIH have been modified several times over the past decades. The current diagnostic criteria from Friedman et al. are based on a modified version of the Dandy criteria [1]. According to these criteria the presence papilledema; an unremarkable neurological examination except for cranial nerve disorders (especially paresis of the abducens); a cMRT without structural lesions, signs of hydrocephalus or meningeal enhancement; physiological CSF composition; and opening pressure of $\geq 25 \mathrm{~cm}$ CSF in adults and $\geq 8 \mathrm{~cm}$ CSF in children (in non-sedated children of normal weight $\geq 25 \mathrm{~cm}$ ) are required for the diagnosis of IIH [1]. A novelty in the current version of the diagnostic criteria is the possibility to diagnose IIH even in the absence of papilledema if uni- or bilateral abducens paresis is present. If both, papilledema and abducens paresis are absent, a definitive diagnosis is not possible, however a probable diagnosis can still be made if cMRT reveals an empty sella, a flattened posterior aspect of the optic globe, a distended perioptic subarachnoid space (with or without a tortuous optic nerve) and a transverse sinus stenosis [1].

Nevertheless, leaving the diagnostic criteria aside, the clinical presentation is characterized primarily by headache, impaired vision and a number of other symptoms including cranial nerve palsies, olfactory disturbances, cognitive deficits, and tinnitus.

\section{Headache}

Up to $90 \%$ of IIH patients suffer from headache, which is the most commonly reported symptom and the main impetus for the initial medical consultation [7, 22]. IIH-associated headache is defined in the diagnostic criteria of the International Headache Society (ICHD 3 beta) [23]. A definitive diagnosis requires an elevated opening pressure of $>25 \mathrm{~cm}$ CSF concurrent with headache that meets two of the three following criteria: Headache has developed in temporal relation to IIH or led to its discovery, headache is relieved by reducing intracranial hypertension, and headache is aggravated in temporal relation to increase in intracranial pressure. In contrast to the 2004 headache criteria (ICHD-2), the current criteria no longer require headache relief after a reduction in intracranial pressure [23]. This change was absolutely essential in light of the fact that many patients can continue to suffer from chronic headache for a long time after pressure has been reduced and intracranial pressure normalized [24].

The clinical presentation of IIH-associated headache is extremely variable, which is why the clinical characteristics of headache were removed from the diagnostic criteria and mentioned only briefly in the comments [23]. The headache is typically described as a bilateral frontal or retro-orbital pressure and occurs almost 
daily [23, 24]. Patients often report worsening with coughing, straining, sneezing or physical exertion [24]. The headache can also be unilateral, have a throbbing character and may even be accompanied by nausea as well as photo- and phonophobia, making it very difficult to differentiate it from other primary headache disorders such as chronic migraines [7, 24, 25].

\section{Visual disorders}

IIH patients frequently suffer from visual disorders. These are primarily caused by pressure-induced papilledema, which can be identified in the overwhelming majority of patients and may be localized uni- or bilaterally [7, 26-28]. Given that IIH can occur without concomitant papilledema, it has been removed as a mandatory diagnostic criteria in the new IIH diagnostic criterion [1] and in those for IIH-associated headache (ICHD C beta) [23].

Papilledema induced by chronic intracranial pressure is usually progressive and causes numerous visual disorders. The most frequently reported visual disorders include obscurations, an enlarged blind spot, an inferior arcuate visual field defect, photopsias, and a loss of visual acuity [7, 27]. As the accompanying papilledema, which is their main underlying cause, visual disturbances are progressive and can cause irreversible deficits that may lead to complete blindness if effective pressure-reducing treatment is not initiated as soon as possible. Pathophysiologically, the chronic increase in pressure in the CSF space leads to an elevated pressure in the optic nerve sheaths resulting in an abnormally high pressure on the optic nerve tissue. This increased pressure reduces the pressure gradient between the intraocular and CSF pressure, which physiologically proceeds centripetally from the eye $[29,30]$. This decreases axoplasmic transport, which in turn causes axonal swelling in the intrabulbar segment of the optic nerve [30]. The retrobulbar segment, where the nerve fibers are myelinated, is not affected. A prelaminar compression of the small, especially venous, capillaries develops secondarily to this axonal swelling. These alterations can also lead to other phenomena such as small hemorrhages in the area of the papilla. Ultimately the changes described cause atrophy in the papilla and irreversible loss of optic nerve function [30]. Imaging techniques can reliably identify both the macroscopic and the microstructural changes resulting from the increased pressure in the area of the optic nerve sheaths [31-33].

No correlation has been observed between the degree of papilledema and the headache [34]. This may be due to the physiological narrowness of the connection between the intracranial subarachnoid space and the optic nerve sheaths, which is also deemed responsible for the occasional several-day latency between acute increases in intracranial pressure and the development of papilledema [30]. Moreover, intracranial pressure is subject to physiological changes over the course of a day, which cannot have short-term effects on the optic nerves owing to the aforementioned narrowness. Even if this would be the case, it would take a longer amount of time to influence axonal transport and cause visual consequences. Nonetheless there are signficant correlations with longer-term parameters. For example, the severity of the papilledema and loss of visual acuity correlate with the incidence of therapy failure [35]. The same is true for evidence of hemorrhages in the area of the papilla, which can already be present with mild visual impairment [36].
In light of the frequency and potential irreversibililty of the visual deficits and their serious long-term impact on quality of life, the diagnostic work-up for a suspected IIH should always include a comprehensive ophthalmological assessment with funduscopy and perimetry. There has been increasing discussion over recent years as to the value of optical coherence tomography (OCT) in diagnosing $\mathrm{IH}$. OCT is a non-invasive procedure that enables, among other things, objective, high-resolution measurement of the thickness of the retinal nerve fiber layer (RFNL). The application of OCT in IIH appears very attractive at first, especially to monitor progress, which can prove useful in individual cases $[37,38]$. An OCT measurement, however, says nothing about the condition of the tissue. For example, in papilledema an initially measured thickening of the RFNL can normalize over the course of the disease, yet it remains unclear whether this normalization is due to a decrease in axonal swelling or the development of papillar atrophy [39]. OCT can therefore augment an ophthalmological examination with funduscopy and perimetry, but certainly not replace it.

In addition to the diagnostic procedures cited, transorbital sonographic measurement of the diameter of the optic nerve sheaths is becoming more important in the diagnosis of intracranial hypertension, because this procedure offers another non-invasive and rapid method for identifying distended optic nerve sheaths and thus supports diagnosis [40-43]. To obtain reliable results, however, the examiner must have extensive experience with this procedure. Due to the relatively low specificity of the procedure, a transorbital sonographic examination is no substitute for a funduscopic examination and even less for the other diagnostic tests (imaging, CSF pressure measurement).

\section{Cranial nerve palsies}

Cranial nerve palsies are observed relatively frequently with IIH. In most cases the abducens nerve is affected, yet palsies may also occur in the oculomotor [44], trochlear [45], trigeminal [46] or facial [47] nerves, or even simultaneously in multiple cranial nerves [48]. The cranial nerve palsies usually abate after effective treatment of the elevated CSF pressure $[47,49]$, so the overall prognosis for these attendant symptoms is commonly good.

Abducens palsy is always accompanied by horizontal double vision and can occur uni- or bilaterally, as is the case in the other cranial nerve palsies. Abducens palsy occurs in nearly $10-20 \%$ of adult cases of $\mathrm{IIH}$ [50], yet its incidence in children could be significantly higher [51]. IIH patients with an attendant abducens palsy more frequently tend to have a higher BMI and a higher CSF pressure compared to patients without cranial nerve palsy [51]. It is not exactly clear why abducens nerve palsy develops in IIH, but it is speculated that the brainstem suffers a discrete downward displacement due to the chronic increase in intracranial pressure, which puts traction on Dorello's canal near the abducens bridge, damaging the nerves [51]. This pathomechanism could also adequately explain why abducens paresis frequently occurs bilaterally in IIH. Similar mechanisms are assumed to underlie the other IIH-associated cranial nerve alterations.

\section{Olfactory dysfunction}

Olfactory dysfunction in IIH has been known for several years $[52,53]$. Approximately $80 \%$ of IIH patients suffer from hyposmia, 
although it is usually mild or moderate, which is probably why it was overlooked for so long. In a clinical study with $17 \mathrm{IIH}$ patients and 17 age- and sex-matched healthy controls, Kunte et al. systematically investigated the occurrence of olfactory impairment in IIH using so-called "sniffin sticks". This test measures odor threshold, discrimination, and identification and can be used to assess them individually and to calculate a TDI score. The study was able to demonstrate that odor discrimination and identification were significantly impaired in the majority of patients. The authors also demonstrated that the patients most affected were those diagnosed within the prior three months and who experienced significant clinical deterioration in those three months. This subgroup also evidenced a reduced odor threshold [53].

The underlying mechanisms have yet to be elucidated. Nonetheless, structural changes in the olfactory nerve are known to occur [54]. In this regard, it was shown that IIH patients, especially those in an early stage of the disease, had reduced olfactory bulb volume [54]. This is most probably the structural correlate for the frequently verifiable functional deficit [55-57]. It remains unclear whether the reduced olfactory bulb volume is simply the direct result of the increased intracranial pressure. The fact that relieving pressure through a lumbar puncture considerably improves olfactory function in a short time suggests that this may be the case [58].

\section{Tinnitus}

IIH patients frequently report uni- or bilateral pulsatile tinnitus [7]. The underlying cause of this alteration remains unclear. The most popular hypothesis assumes that pulsatile tinnitus could arise from one or more TSS. It is postulated that audible turbulences in the blood flow occur due to the vascular narrowing. This hypothesis is supported by the fact that invasive elimination of the stenosis using an intravascular stent frequently resolves the tinnitus. In a prospective study of $29 \mathrm{IIH}$ patients with TSS and pulsatile tinnitus who were treated with a stent placed in the transverse sinus, Boddu et al. demonstrated that in all treated patients the tinnitus remitted on the day of the intervention. During the two-year follow-up phase, $10 \%$ of patients were restenosed when the tinnitus reappeared. Even though these data offer strong support for a causal relationship, other studies must be conducted to validate this pathophysiological connection especially because the number of $\mathrm{IIH}$ patients with stenosis who experience pulsatile tinnitus is unknown as is the number of IIH patients with tinnitus who have a TSS.

\section{Cognitive deficits}

Indications of cognitive deficits in IIH have been increasing for several years. The assumption was first based on case reports and small clinical studies in which individual cognitive domains were examined [59-61]. In a prospective study, Yri et al. showed that patients with IIH frequently suffer from a global cognitive dysfunction. The most severe deficits were found in processing speed and reaction time [62]. The exact mechanism that causes the identified deficits remains unclear. The deficits could conceivably be the direct result of the increased intracranial pressure, however even after intracranial pressure is normalized through adequate treatment, the cognitive deficits commonly persist at least at the three-month fol- low-up [62]. Therefore, some evidence points to cognitive deficits having more complex and long-lasting causes than simple mechanical compression from increasing intracranial pressure. Because patients with IIH often experience a depressed mood during their illness, which in itself can impair cognitive function, it cannot be ruled out that this kind of connection also underlies the described association. In spite of the currently available data, however, further prospective studies with longer follow-up phases are urgently needed, because the cognitive deficits significantly reduce the quality of life and are an essential burden for social participation and occupational reintegration. For these reasons, and especially because it remains unclear if the deficits are reversible, a diagnostic evaluation of IIH should also include neuropsychological testing for early detection of cognitive dysfunction.

\section{CSF diagnostics}

The clinical work-up for IIH requires a lumbar puncture to measure the opening CSF pressure. Not only should the opening pressure be measured, but the CSF composition should be analyzed to rule out any secondary causes. To prevent any influence on the measurements, pressure is measured with the patient in supine position and in an unsedated state. According to the current diagnostic criteria, an opening pressure of $\geq 25 \mathrm{~cm}$ CSF in adults $\geq 28 \mathrm{~cm}$ CSF in children is considered pathological [1]. CSF pressure can vary widely, even during the course of a day. If there is a justifiable suspicion of a PTC, a second lumbar puncture should be considered under certain circumstances depending on clinical symptoms [63]. Temporary continuous pressure measurement may be necessary in rare and justified cases $[64,65]$. The CSF composition should be unremarkable [1].

\section{Magnetic resonance imaging (MRI)}

MRI has gained increasing importance in diagnosing IIH over the last several years and should be an essential component of the diagnostic evaluation. First it is required to rule out diseases that can cause a secondary increase in intracranial pressure as well as to identify IIH-specific changes. The most significant IIH-associated changes in a cerebral MRI include an empty sella, a flattened posterior aspect of the optic globe, a distended perioptic subarachnoid space with or without a tortuous optic nerve, and the presence of uni- or bilaterally localized TSS [1,33, 66-68]. Based on the fact that TSS are verifiable in up to $90 \%$ of $\mathrm{IIH}$ patients and may play a causative role, an MR venography (MRV) should also be conducted in addition to a structural MRI [19]. The MRV not only allows to identify a TSS but more importantly it can also rule out sinus or cranial vein thromboses. This is particularly signficant because thromboses can also occur as clinically inapparent microthromboses that could still impede venous drainage and thus increase intracranial pressure and, in some circumstances, lead to further secondary TSS from the external pressure [20]. It is not yet clear whether TSS is the cause or the consequence of intracranial hypertension.

To sum up, however, it should be noted that changes identified in the MRI can substantially support the diagnosis of an IIH but due to their sensitivity and specificity current MR techniques can by no means replace lumbar puncture and measurement of the CSF opening pressure. 


\section{Treatment}

The principal aims of IIH treatment are the preservation of visual acuity and the relief of headache. Treatment should always follow a multimodal approach consisting primarily of effective weight loss augmented by pharmacological treatment with carboanhydrase inhibitors. Invasive therapeutic procedures such as fenestration of the optic nerve sheaths, endovascular procedures and shunt systems should be used only in treatment-resistant cases, especially if the risk of visual loss is significant.

\section{Weight loss}

Considering the highly significant correlation between obesity and chronic intracranial hypertension, weight loss should always be the main component of treatment. Numerous studies have now shown that weight loss significantly reduces intracranial pressure, papilledema, the associated visual disturbances and headache [69]. Even small changes can have a major influence on the course of the disease. Interestingly, this is also true for patients whose weight is initially normal [8]. Successful weight loss must always be carefully monitored to ensure it is maintained, because regaining of weight is associated with an increased recurrence of IIH [70]. If weight loss cannot be maintained in the severely obese, surgical intervention through bariatric surgery should be considered, although the invasivity of this procedure warrants restricting it to treatment-resistant cases [71].

\section{Lumbar puncture}

The lumbar puncture is the essential component of the diagnostic workup of IIH. Due to its invasiveness, however, its importance in the treatment of IIH is only secondary. It can still be considered as a bridging measure prior to an endovascular or surgical intervention in treatment-resistant situations where vision loss is imminent.

The sudden drop in intracranial pressure induced by the lumbar puncture usually temporarily relieves the headache, although strictly speaking this observation has not yet been proven in any prospective clinical study. The same is true for the effect of the acute drop in pressure on other IIH-associated symptoms. Interestingly, the lessening of headache intensity in some IIH patients lasts for several weeks, although the amount of fluid drained is replaced after a few hours [72]. Even a puncture-induced leak persisting several days cannot sufficiently explain this observation. It is feasible, however, that relieving the pressure reestablishes a stable equilibrium between CSF production and absorption, thus reducing secondary pressure-induced TSS and thereby improving venous drainage and CSF absorption [73-76]. Further studies are needed to confirm this hypothesis.

\section{Pharmaceutical treatment}

Carboanhydrase inhibitors form the first-line pharmaceutical treatment of IIH. Carboanhydrase is localized in the choroid plexus and regulates the production of hydrogen carbonate $\left(\mathrm{HCO}_{3}{ }^{-}\right)$and hence CSF secretion [77]. Inhibiting this enzyme reduces CSF production [78] and therefore reduces CSF pressure [79].

Acetazolamide is the most frequently used carboanhydrase inhibitor in the treatment of $\mathrm{IH}$, and the typical dose range is between 500-2000 mg. Although administered for many years to treat $\mathrm{IIH}$, until recently the use acetazolamide had been based on the results of open or uncontrolled studies without a placebo group $[80,81]$. The first randomized placebo-controlled study on the efficacy of acetazolamide in treating $\mathrm{IIH}$ was conducted just a few years ago. The study showed that acetazolamide, at the study dose of up to $4 \mathrm{~g}$ daily, improved papilledema, visual deficits, and headache [82]. In spite of the relatively frequent gastrointestinal side effects, the treatment significantly improved quality of life [83]. This clinical improvement in all the aspects cited continued throughout a follow-up period of 12 months [84]. Acetazolamide brought about significant weight loss in the study, which probably contributed substantially to its efficacy $[82,85]$.

In cases where acetazolamide is not effective, tolerated, or contraindicated, topiramate can be given as an alternative at a dose between 50-200 mg. To date, however, there have been no randomized, placebo-controlled studies to verify its efficacy. Only a few open studies without a placebo group suggest its efficacy in $\mathrm{IIH}$ $[80,86]$. Paresthesias, mood swings, and cognitive deficits are among the most frequent side effects [87]. The essential mode of action is thought to be the inhibition of the carboanhydrase. Topiramate frequently also leads to significant weight loss, however, thus likely substantially contributing to its efficacy.

Furosemide is yet another pharmaceutical option. Furosemide also inhibits carboanhydrase, thereby reducing CSF production and hence CSF pressure [88]. The dosage range for the treatment of IIH is usually between 30 and $80 \mathrm{mg}$ a day. There have been no randomized, placebo-controlled studies on its efficacy in the treatment of IIH to date.

Besides the carboanhydrase inhibitors described, octreotide is showing promise for use in IIH. This drug is a long-acting, synthetic somatostatin analog that also inhibits the effect of growth hormone $(\mathrm{GH})$ and insulin-like growth factor 1 (IGF-1). Existing data suggest that octreotide could considerably improve papilledema and the associated visual deficits as well as the headache [89-92]. Its efficacy thereby extends far beyond its known analgesic effect [93]. Its precise mode of action in IIH is unknown. The idea to use octreotide originated from the observation that treatment with $\mathrm{GH}$ or IGF-1 could trigger a secondary PTC $[90,94]$. However, the patients treated in the three cited studies suffered from IIH, i. e., there were no pathological hormonal changes present. All studies were open studies or case reports, however, so further studies to test its efficacy are essential.

\section{Endovascular treatment}

An endovascular stent can be an effective treatment option in the case of one or more verified stenoses in the transverse sinus $[18,20]$. The specific mode of action remains unclear, but it is assumed that eliminating the TSS improves venous drainage, which in turn increases CSF absorption, ultimately reducing CSF pressure $[95,96]$. In spite of the relatively good data on its efficacy, endovascular treatment should be considered only in treatment-resistant cases. For one thing, the long-term data on efficacy and safety is limited. Furthermore, complications such as stent displacement, in-stent thrombosis, or sinus perforation with consecutive subdural hematoma are not uncommon [97]. 


\section{Fenestration of the optical nerve sheaths}

This invasive procedure consists of an incision in the meninges surrounding the optical nerve. The mode of action has not yet been elucidated. It is assumed that the incision reduces the CSF pressure around the optic nerve, but it is not clear why this effect persists even though the meningeal lesion heals after a short time, thereby eliminating the CSF leak. Numerous studies have already proven the efficacy of fenestration in reducing papilledema and its associated visual deficits, however data on its long-term efficacy and safety are insufficient $[98,99]$. This intervention should therefore be considered only in particular treatment-resistant cases where the risk of irreversible vision loss is high [6].

\section{CSF diversion procedures}

A shunt system aims to reduce pressure by circumventing the physiological CSF drainage pathway. Either a ventriculoperitoneal or a lumboperitoneal shunt can be used, whereby the former is preferable because it is associated with a lesser risk of side effects and necessary shunt revisions [100]. Nonetheless, potentially dangerous side effects such as shunt infection or dysfunction can occur with the procedure. Furthermore, data on long-term efficacy and safety are sparse [101]. This data does suggest that in most cases placement of a shunt system positively affects the visual deficits but not the headache. Furthermore, for a variety of reasons more than half of the patients require a shunt revision [101,102]. Against this backdrop, this intervention should be considered only in treatment-resistant patients with a significant risk of irreversible vision loss.

\section{Conflict of interest}

J. Hoffmann received fees for consulting, and/or other advisory board activities from Allergan, Autonomic Technologies Inc. (ATI), Chordate Medical and Novartis. He also received fees for lecturing activities from Allergan, Novartis and Teva. There is no conflict of interest with respect to the content of this manuscript. In the last two years, A. May has received and/or continues to receive funding from the German Research Association (DFG), the Federal Ministry of Education and Research (BMBF), and the European Union. He also has an unrestricted grant from Chordate Medical. In the last two years he has been a consultant or speaker for Allergan, Autonomic Technologies Inc. (ATI), ElectroCore, TEVA and Desitin. There is no conflict of interest with respect to the content of this manuscript.

\section{References}

[1] Friedman DI, Liu GT, Digre KB. Revised diagnostic criteria for the pseudotumor cerebri syndrome in adults and children. Neurology 2013; 81: 1159-1165

[2] Friedman DI. The pseudotumor cerebri syndrome. Neurol Clin 2014; 32: 363-396

[3] Durcan FJ, Corbett J], Wall M. The incidence of pseudotumor cerebri. Population studies in lowa and Louisiana. Arch Neurol 1988; 45: 875-877
[4] Radhakrishnan K, Ahlskog JE, Cross SA et al. Idiopathic intracranial hypertension (pseudotumor cerebri). Descriptive epidemiology in Rochester, Minn, 1976 to 1990. Arch Neurol 1993; 50: 78-80

[5] Hoffmann ], Goadsby PJ. Update on intracranial hypertension and hypotension. Curr Opin Neurol 2013; 26: 240-247

[6] Hoffmann J. Headache Attributed to Intracranial Hypertension and Hypotension. In: Mitsikostas DD, Pameleire K, Hrsg. Pharmacological Management of Headaches. 1. Aufl. Springer; 2015: 189-205

[7] Wall M, Kupersmith M], Kieburtz KD et al. The idiopathic intracranial hypertension treatment trial: Clinical profile at baseline. JAMA Neurol 2014; 71: 693-701

[8] Daniels AB, Liu GT, Volpe NJ et al. Profiles of obesity, weight gain, and quality of life in idiopathic intracranial hypertension (pseudotumor cerebri). Am J Ophthalmol 2007; 143: 635-641

[9] Almarzouqi S], Morgan ML, Lee AG. Idiopathic intracranial hypertension in the Middle East: A growing concern. Saudi J Ophtamlol 2015; 29: $26-31$

[10] Wall M. Idiopathic intracranial hypertension. Neurol Clin 2010; 28 : 593-617

[11] Ko MW, Liu GT. Pediatric idiopathic intracranial hypertension (pseudotumor cerebri). Horm Res Paediatr 2010; 74: 381-389

[12] Cinciripini GS, Donahue S, Borchert MS. Idiopathic intracranial hypertension in prepubertal pediatric patients: Characteristics, treatment, and outcome. Am J Ophthalmol 1999; 127: 178-182

[13] Sheldon CA, Paley GL, Xiao R et al. Pediatric idiopathic intracranial hypertension: Age, gender, and anthropometric features at diagnosis in a large, retrospective, multisite cohort. Ophthalmology 2016; 123 : 2424-2431

[14] Mendelson CR, Simpson ER. Regulation of estrogen biosynthesis by human adipose cells in vitro. Mol Cell Endocrinol 1987; 52: 169-176

[15] Markey KA, Uldall M, Botfield $\mathrm{H}$ et al. Idiopathic intracranial hypertension, hormones, and 11beta-hydroxysteroid dehydrogenases. J Pain Res 2016; 9: 223-232

[16] Ball AK, Sinclair AJ, Curnow S] et al. Elevated cerebrospinal fluid (CSF) leptin in idiopathic intracranial hypertension (IIH): Evidence for hypothalamic leptin resistance? Clin Endocrinol (Oxf) 2017; 37: 113-121

[17] Lampl Y, Eshel Y, Kessler A et al. Serum leptin level in women with idiopathic intracranial hypertension. J Neurol Neurosurg Psychiatry 2002; 72: 642-643

[18] Dinkin M], Patsalides A. Venous sinus stenting in idiopathic intracranial hypertension: Results of a prospective trial. J Neuroophthalmol 2017; 37: 113-121

[19] Farb RI, Vanek I, Scott JN et al. Idiopathic intracranial hypertension: the prevalence and morphology of sinovenous stenosis. Neurology 2003; 60: 1418-1424

[20] Ahmed RM, Wilkinson M, Parker GD et al. Transverse sinus stenting for idiopathic intracranial hypertension: a review of 52 patients and of model predictions. AJNR Am J Neuroradiol 2011; 32: 1408-1414

[21] Bedford THB. The effect of increased intracranial venous pressure on the pressure of the cerebrospinal fluid. Brain 1935; 58: 427-447

[22] Giuseffi V, Wall M, Siegel PZ et al. Symptoms and disease associations in idiopathic intracranial hypertension (pseudotumor cerebri): A case control study. Neurology 1991; 41: 239

[23] Headache classification committee of the international headache society. The international classification of headache disorders, 3rd edition (beta version). Cephalalgia 2013; 33: 629-808

[24] Yri HM, Rönnbäck C, Wegener M et al. The course of headache in idiopathic intracranial hypertension: A 12-month prospective follow-up study. Eur J Neurol 2014; 21: 1458-1464

[25] Wall M. The headache profile of idiopathic intracranial hypertension. Cephalalgia 1990; 10: 331-335 
[26] Wall M, White WN 2nd. Asymmetric papilledema in idiopathic intracranial hypertension: Prospective interocular comparison of sensory visual function. Invest Ophthalmol Vis Sci 1998; 39: 134-142

[27] Keltner JL, Johnson CA, Cello KE et al. Baseline visual field findings in the Idiopathic Intracranial Hypertension Treatment Trial (IIHTT). Invest Ophthalmol Vis Sci 2014; 55: 3200-3207

[28] Bidot S, Bruce BB, Saindane AM et al. Asymmetric papilledema in idiopathic intracranial hypertension. J Neuroophthalmol 2015; 35: 31-36

[29] Jonas JB, Ritch R, Panda-Jonas S. Cerebrospinal fluid pressure in the pathogenesis of glaucoma. In: Giacinto B, Carlo N, eds. New Trends in Basic and Clinical Research of Glaucoma: A Neurodegenerative Disease of the Visual System Part B (Progress in Brain Research 225). Amsterdam, Elsevier 2015: 33-47

[30] Hayreh SS. Pathogenesis of optic disc edema in raised intracranial pressure. Prog Retin Eye Res 2016; 50: 108-144

[31] Schmidt C, Wiener E, Lüdemann L et al. Does IIH alter brain microstructures? - A DTI-based approach. Headache: 2017; 57: 746-755

[32] Hoffmann J, Schmidt C, Kunte H et al. Volumetric assessment of optic nerve sheath and hypophysis in idiopathic intracranial hypertension. AJNR Am J Neuroradiol 2014; 35: 513-518

[33] Hoffmann J, Huppertz H], Schmidt C et al. Morphometric and volumetric MRI changes in idiopathic intracranial hypertension. Cephalalgia 2013; 33: 1075-1084

[34] Digre KB, Nakamoto BK, Warner JE et al. A comparison of idiopathic intracranial hypertension with and without papilledema. Headache 2009; 49: 185-193

[35] Wall M, Falardeau J, Fletcher WA et al. Risk factors for poor visual outcome in patients with idiopathic intracranial hypertension. Neurology 2015; 85: 799-805

[36] Wall M, Thurtell M]. Optic disc haemorrhages at baseline as a risk factor for poor outcome in the idiopathic intracranial hypertension treatment trial. Br j Ophthalmol 2017 doi:10.1136/bjophthalmol-2016-309852

[37] Kaufhold F, Kadas EM, Schmidt C et al. Optic nerve head quantification in idiopathic intracranial hypertension by spectral domain OCT. PLoS One 2012; 7: e36965

[38] OCT Sub-Study Committee for the Nordic Idiopathic Intracranial Hypertension Study. Baseline OCT Measurements in the Idiopathic Intracranial Hypertension Treatment Trial, Part I: Quality Control, Comparisons, and VariabilityBaseline OCT Measurements in IIHTT, Part I. Invest Ophthalmol Vis Sci 2014; 55: 8180-8188

[39] Rebolleda G, Muñoz-Negrete F]. Follow-up of mild papilledema in idiopathic intracranial hypertension with optical coherence tomography. Invest Ophthalmol Vis Sci 2009; 50: 5197-5200

[40] Lochner P, Brigo F, Zedde ML et al. Feasibility and usefulness of ultrasonography in idiopathic intracranial hypertension or secondary intracranial hypertension. BMC Neurology 2016; 16: 85

[41] Moretti R, Pizzi B, Cassini F et al. Reliability of optic nerve ultrasound for the evaluation of patients with spontaneous intracranial hemorrhage. Neurocrit Care 2009; 11: 406-410

[42] Geeraerts T, Merceron S, Benhamou D et al. Non-invasive assessment of intracranial pressure using ocular sonography in neurocritical care patients. Intensive Care Med 2008; 34: 2062-2067

[43] Bäuerle J, Nedelmann M. Sonographic assessment of the optic nerve sheath in idiopathic intracranial hypertension. J Neurol 2011; 258: 2014-2019

[44] McCammon A, Kaufman HH, DSears ES. Transient oculomotor paralysis in pseudotumor cerebri. Neurology 1981; 31: 182-184

[45] Lee AG. Fourth nerve palsy in pseudotumor cerebri. Strabismus 1995; 3: 57-59
[46] Davenport RJ, Will RG, Galloway PJ. Isolated intracranial hypertension presenting with trigeminal neuropathy. J Neurol Neurosurg Psychiatry 1994; 57: 381

[47] Capobianco DJ, Brazis PW, Cheshire WP. Idiopathic intracranial hypertension and seventh nerve palsy. Headache 1997; 37: 286-288

[48] Soroken C, Lacroix L, Korff CM. Combined VIth and VIIth nerve palsy: Consider idiopathic intracranial hypertension! Eur J Paed Neurol 2016; 20: 336-338

[49] Davie C, Kennedy P, Katifi HA. Seventh nerve palsy as a false localising sign. J Neurol Neurosurg Psychiatry 1992; 55: 510-511

[50] Wall M, George D. Idiopathic intracranial hypertension. A prospective study of 50 patients. Brain 1991; 114 (Pt 1A): 155-180

[51] Reid JE, Reem RE, Aylward SC et al. Sixth nerve palsy in paediatric intracranial hypertension. Neuro-ophthalmology 2016; 40: 23-27

[52] Khoo KF, Kunte H. Olfactory dysfunction in patients with idiopathic intracranial hypertension. Neurology 2014; 82: 189

[53] Kunte H, Schmidt F, Kronenberg G et al. Olfactory dysfunction in patients with idiopathic intracranial hypertension. Neurology 2013; 81: 379-382

[54] Schmidt C, Wiener E, Hoffmann J et al. Structural olfactory nerve changes in patients suffering from idiopathic intracranial hypertension. PLoS One 2012; 7: e35221

[55] Goektas O, Fleiner F, Sedlmaier B et al. Correlation of olfactory dysfunction of different etiologies in MRI and comparison with subjective and objective olfactometry. Eur J Radiol 2009; 71 : 469-473

[56] Hummel T, Urbig A, Huart C et al. Volume of olfactory bulb and depth of olfactory sulcus in 378 consecutive patients with olfactory loss. J Neurol 2015; 262: 1046-1051

[57] Buschhüter D, Smitka M, Puschmann S et al. Correlation between olfactory bulb volume and olfactory function. Neuroimage 2008; 42 : 498-502

[58] Becker N, Kronenberg G, Harms L et al. Rapid improvement of olfaction after lumbar puncture in a patient with idiopathic intracranial hypertension. Headache 2016 doi: 10.1111/head.12802

[59] Kaplan CP, Miner ME, McGregor JM. Pseudotumour cerebri: Risk for cognitive impairment? Brain Inj 1997; 11: 293-303

[60] Sorensen PS, Thomsen AM, Gjerris F. Persistent disturbances of cognitive functions in patients with pseudotumor cerebri. Acta Neurol Scand 1986; 73: 264-268

[61] Kharkar S, Hernandez R, Batra S et al. Cognitive impairment in patients with pseudotumor cerebri syndrome. Behav Neurol 2011; 24: $143-148$

[62] Yri HM, Fagerlund B, Forchhammer HB et al. Cognitive function in idiopathic intracranial hypertension: A prospective case-control study. BMJ Open 2014; 4: e004376

[63] Bono F, Salvino D, Tallarico T et al. Abnormal pressure waves in headache sufferers with bilateral transverse sinus stenosis. Cephalalgia 2010; 30: 1419-1425

[64] Torbey MT, Geocadin RG, Razumovsky AY et al. Utility of CSF pressure monitoring to identify idiopathic intracranial hypertension without papilledema in patients with chronic daily headache. Cephalalgia 2004; 24: 495-502

[65] Toma AK, Tarnaris A, Kitchen ND et al. Continuous intracranial pressure monitoring in pseudotumour cerebri: Single centre experience. $\mathrm{Br}$ J Neurosurg 2010; 24: 584-588

[66] Agid R, Farb RI, Willinsky RA et al. Idiopathic intracranial hypertension: The validity of cross-sectional neuroimaging signs. Neuroradiology 2006; 48: 521-527

[67] Degnan AJ, Levy LM. Pseudotumor Cerebri: Brief review of clinical syndrome and imaging findings. AJNR Am J Neuroradiol 2011; 32: 1986-1993 
[68] Maralani PJ, Hassanlou M, Torres C et al. Accuracy of brain imaging in the diagnosis of idiopathic intracranial hypertension. Clin Radiol 2012; 67: 656-663

[69] Sinclair A], Burdon MA, Nightingale PG et al. Low energy diet and intracranial pressure in women with idiopathic intracranial hypertension: prospective cohort study. BMJ 2010; 341: c2701

[70] Ko MW, Chang SC, Ridha MA et al. Weight gain and recurrence in idiopathic intracranial hypertension: A case-control study. Neurology 2011; 76: 1564-1567

[71] Manfield JH, Yu KK-H, Efthimiou E et al. Bariatric surgery or non-surgical weight loss for idiopathic intracranial hypertension? a systematic review and comparison of meta-analyses. Obesity Surgery 2017; 27: 513-521

[72] Johnston I, Paterson A. Beningn intracranial hypertension: II. CSF pressure and and circulation. Brain 1974; 97: 301-312

[73] Biousse V, Bruce BB, Newman NJ. Update on the pathophysiology and management of idiopathic intracranial hypertension. J Neurol Neurosurg Psychiatry 2012; 83: 488-494

[74] Scoffings DJ, Pickard JD, Higgins JN. Resolution of transverse sinus stenoses immediately after CSF withdrawal in idiopathic intracranial hypertension. J Neurol Neurosurg Psychiatry 2007; 78: 911-912

[75] Bateman GA, Stevens SA, Stimpson J. A mathematical model of idiopathic intracranial hypertension incorporating increased arterial inflow and variable venous outflow collapsibility. J Neurosurg 2009; 110: $446-456$

[76] De Simone R, Marano E, Fiorillo C et al. Sudden re-opening of collapsed transverse sinuses and longstanding clinical remission after a single lumbar puncture in a case of idiopathic intracranial hypertension. Pathogenetic implications. Neurol Sci 2005; 25: 342-344

[77] Damkier HH, Brown PD, Praetorius ]. Cerebrospinal fluid secretion by the choroid plexus. Physiol Rev 2013; 93: 1847-1892

[78] Rubin RC, Henderson ES, Ommaya AK et al. The production of cerebrospinal fluid in man and its modification by acetazolamide. J Neurosurg 1966; 25: 430-436

[79] Gucer G, Viernstein L. Long-term intracranial pressure recording in the management of pseudotumor cerebri. J Neurosurg 1978; 49: 256-263

[80] Celebisoy N, Gokcay F, Sirin H et al. Treatment of idiopathic intracranial hypertension: Topiramate vs acetazolamide, an open-label study. Acta Neurol Scand 2007; 116: 322-327

[81] Ball AK, Howman A, Wheatley $\mathrm{K}$ et al. A randomised controlled trial of treatment for idiopathic intracranial hypertension. J Neurol 2011; 258: 874-881

[82] Wall M, McDermott MP, Kieburtz KD et al. Effect of acetazolamide on visual function in patients with idiopathic intracranial hypertension and mild visual loss: The idiopathic intracranial hypertension treatment trial. JAMA 2014; 311: 1641-1651

[83] Digre KB, Bruce BB, McDermott MP et al. Quality of life in idiopathic intracranial hypertension at diagnosis: IIH Treatment Trial results. Neurology 2015; 84: 2449-2456

[84] Wall M, Kupersmith M], Thurtell M] et al. The longitudinal idiopathic intracranial hypertension trial: outcomes from months $6-12$. Am J Ophthalmol 2017; 176: 102-107
[85] Sinclair A], Woolley R, Mollan SP. Idiopathic intracranial hypertension. JAMA 2014; 312: 1059-1060

[86] Shah VA, Fung S, Shahbaz R et al. Idiopathic intracranial hypertension. Ophthalmology 2007; 114: 617

[87] Loring DW, Williamson DJ, Meador KJ et al. Topiramate dose effects on cognition: A randomized double-blind study. Neurology 2011; 76: 131-137

[88] Carta F, Supuran CT. Diuretics with carbonic anhydrase inhibitory action: A patent and literature review (2005 - 2013). Expert Opin Ther Pat 2013; 23: 681-691

[89] Deftereos SN, Panagopoulos G, Georgonikou D et al. Treatment of idiopathic intracranial hypertension: Is there a place for octreotide? Cephalalgia 2011; 31: 1679-1680

[90] House PM, Stodieck SRG. Octreotide: The IIH therapy beyond weight loss, carbonic anhydrase inhibitors, lumbar punctures and surgical/ interventional treatments. Clin Neurol Neurosurg 2016; 150: 181-184

[91] Panagopoulos GN, Deftereos SN, Tagaris GA et al. Octreotide: a therapeutic option for idiopathic intracranial hypertension. Neurol Neurophysiol Neurosci 2007; 1:

[92] Antaraki A, Piadites G, Vergados ] et al. Octreotide in benign intracranial hypertension. Lancet 1993; 342: 1170

[93] Williams G, Ball JA, Lawson RA et al. Analgesic effect of somatostatin analogue (octreotide) in headache associated with pituitary tumours. Br Med J Clin Res Ed 1987; 295: 247-248

[94] [94] Malozowski S, Tanner LA, Wysowski D et al. Growth hormone, insulin-like growth factor $i$, and benign intracranial hypertension. N Engl J Med 1993; 329: 665-666

[95] Owler BK, Parker G, Halmagyi GM et al. Cranial venous outflow obstruction and pseudotumor Cerebri syndrome. Adv Tech Stand Neurosurg 2005; 30: 107-174

[96] Owler BK, Parker G, Halmagyi GM et al. Pseudotumor cerebri syndrome: venous sinus obstruction and its treatment with stent placement. J Neurosurg 2003; 98: 1045-1055

[97] Lueck C, Mcllwaine G. Interventions for idiopathic intracranial hypertension. Cochrane Database Syst Rev 2005; CD003434

[98] Spoor TC, McHenry JG. Long-term effectiveness of optic nerve sheath decompression for pseudotumor cerebri. Arch Ophthalmol 1993; 111: 632-635

[99] Kelman SE, Heaps R, Wolf A et al. Optic nerve decompression surgery improves visual function in patients with pseudotumor cerebri. Neurosurgery 1992; 30: 391-395

[100] Menger RP, Connor DE Jr., Thakur JD et al. A comparison of lumboperitoneal and ventriculoperitoneal shunting for idiopathic intracranial hypertension: An analysis of economic impact and complications using the Nationwide Inpatient Sample. Neurosurg Focus 2014; 37: E4

[101] Sinclair A], Kuruvath S, Sen D et al. Is cerebrospinal fluid shunting in idiopathic intracranial hypertension worthwhile? A 10-year review. Cephalalgia 2011; 31: 1627-1633

[102] Batra R, Sinclair A. Idiopathic intracranial hypertension; Research progress and emerging themes. J Neurol 2014; 261: 451-460 